\title{
A NEW TECHNIQUE TO DIFFERENTIATE BETWEEN GEMINATE AND NONGEMINATE RECOMBINATION OF TRIPLET EXCITONS
}

\author{
J. PRASAD and R. KOPELMAN \\ Department of Chemistry, The University of Michigan, Ann Arbor, MI 48109-1055, USA
}

\begin{abstract}
Geminate formation and recombination of the triplet excitons in the 2,3-benzocarbazole/tetracene has been studied using a dynamic technique (pulse vs. steady state excitation). In tetracene, the mode of decay of the first excited singlet state is by fission into two neighboring triplet excitons that can undergo geminate recombination. The concentration of 2,3-benzocarbazole in our samples was $20 \%$. We monitored the geminate exciton recombination via delayed fluorescence at room temperature. Decay rates were monitored using steady state and pulsed excitations. Decay rates for the 2,3-benzocarbazole/ tetracene samples were then compared with the decay rates for nongeminate recombination in naphthalene samples. We find that the geminate recombination decay rates do not depend on the duration of the excitation (pulse length). For nongeminate recombination the decay rates were found to depend on the pulse length (duration of excitation). Thus our work clearly provides a new, dynamic technique to differentiate between geminate and nongeminate recombination of triplet excitons.
\end{abstract}

\section{Introduction}

Classically, a steady-state buildup from a random excitation of a material will result in a random (Poisson) distribution of particles. This is true for the creation of defects, of electrons and holes, of solitons and antisolitons, as well as of excitons. We address here the simple case of exciton creation and annihilation (fusion):

$h \nu \rightarrow \mathrm{A} \quad \mathrm{A}+\mathrm{A} \rightarrow h \nu^{\prime}$.

Obviously, an excitation by a random pulse of photons creates a random ensemble of excitons. On the other hand, a steady-state excitation may lead to a nonclassical, non-Poisson particle distribution [1]. This selfordering occurs for fractal-like, low-dimensional or random media (but not for 3-dimensional homogeneous media). An example is given in ref. [2]. However, the exact nature of the source term determines the outcome. It has been pointed out [3] that a geminate creation of excitations should lead to a Poisson (random) steadystate distribution, resulting in "classical kinetics", even for low-dimensional and fractal materials. In this work we test this out experimentally. We selected a system where geminate creation of excitons is the only channel of particle formation. We contrast this with another system, where random creation of single excitons is the sole channel for particle creation. These systems are crystals of tetracene for geminate and naphthalene for nongeminate exciton creation.

The nature of radiationless transitions in organic molecular solids and molecules has been a subject of intensive investigation. A striking example of radiationless processes occurs in tetracene, which is a homolog of anthracene in the linear polyacene aromatic hydro- carbon series. Solid anthracene has a fluorescence efficiency, $\sim 0.95$ [4], whereas for tetracene it is $\sim 0.002$ [5] at $298 \mathrm{~K}$. This is because in crystalline tetracene, at $298 \mathrm{~K}$, the excitation of the singlet exciton results in the thermally activated, spin-allowed production of two triplet excitons with almost unit efficiency. This exciton fission process is a result of an accidental near degeneracy of the energy of the singlet exciton with that of the energy of two triplet excitons. At low temperatures (77 $\mathrm{K})$, the fission process is essentially inhibited. Energetically, this mechanism is feasible in tetracene, but not in anthracene or naphthalene. This constitutes a process of singlet exciton fission, which is the reverse of the mutual annihilation of two triplet excitons to produce one singlet (exciton fusion). Virtually every singlet exciton produced in tetracene fissions into two $T$ excitons [6]. Since these excitons are generated as near neighbors, the local concentration of $\mathrm{T}$ excitons is huge. This situation is different from the case where $T$ excitons are not generated by this fission mechanism. Our studies exploit this difference. The situation is analogous to that of ionization in particle tracks in which positive and negative carriers are produced in close proximity to each other; the local concentration of carriers is very high, although the average concentration in the crystal may be vanishingly small. Thus, fission bears the same relation to excitons as ionization does to carriers. A dominant early process in ionization is geminate recombination, and the same process occurs in the exciton fission process.

To exploit the difference in geminate and nongeminate processes, we applied a new dynamic technique, developed in our laboratory. For geminate recombination study we doped tetracene with 2,3-benzo- 
carbazole $(\mathrm{BC})$ in order to construct a system with $\mathrm{T}$ exciton reflectors [7]. None of the low lying excited electronic states of $\mathrm{BC}$ is accessible from either low-lying excited electronic state of tetracene. The $\mathrm{T}$ energy level of $\mathrm{BC}$ lies at $18200 \mathrm{~cm}^{-1}(2.26 \mathrm{eV})$ [4] which is $1 \mathrm{eV}$ higher than the tetracene $\mathrm{T}$ energy $(1.27 \mathrm{eV})$ and is just below that of the singlet level of tetracene $2.35 \mathrm{eV}$ [6]. The singlet level of $\mathrm{BC}$ lies at $3.2 \mathrm{eV}$ [4], which is thermally inaccessible to the tetracene singlet exciton. Most important, BC was chosen because it forms a solid solution with tetracene.

\section{Experimental}

To prepare the 2,3-benzocarbazole/tetracene mixtures we used the same method as described in ref. [7]. The concentration of 2,3-benzocarbazole in our samples was $20 \%$. For nongeminate studies, we used naphthalene in 1-dimensional systems [8]. We monitored the geminate and nongeminate exciton recombination via delayed fluorescence. Decay rates were monitored using steady state and pulsed excitations from an argon ion laser with an electro-optical modulator for the tetracene/ $\mathrm{BC}$ geminate studies (microsecond triplet lifetimes). A Xenon lamp and shutter arrangement was used for the naphthalene (nongeminate) system where the delayed fluorescence life time extends to seconds.

\section{Results}

Pulsed and steady state decay curves for tetracene/ BC system are shown in fig. 1. As expected, the decay

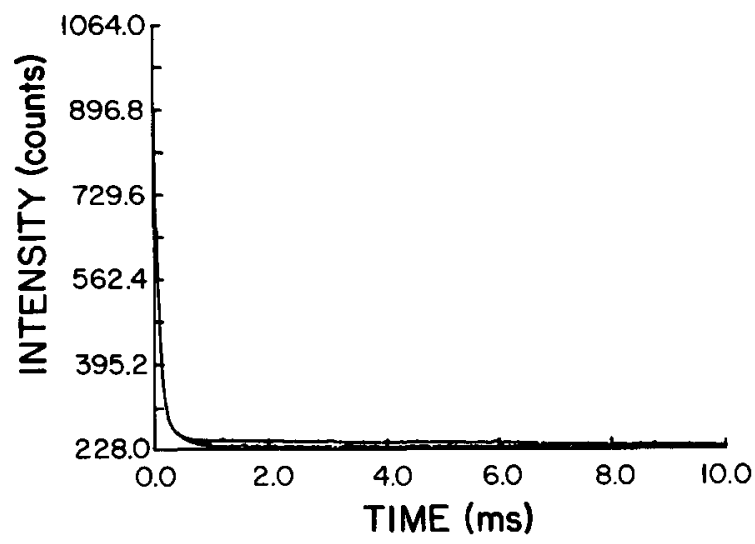

Fig. 1. Decay rates for tetracene/2,3-benzocarbazole (geminate) system. Pulse and steady state conditions were achieved using an electro-optical modulator with argon ion laser (4865 §). In the steady state experiment the sample was excited for $2 \mathrm{~s}$ and then decay rates were monitored. For the pulsed run, $200 \mathrm{~ns}$ pulses were used. The two curves are superimposed (practically indistinguishable).

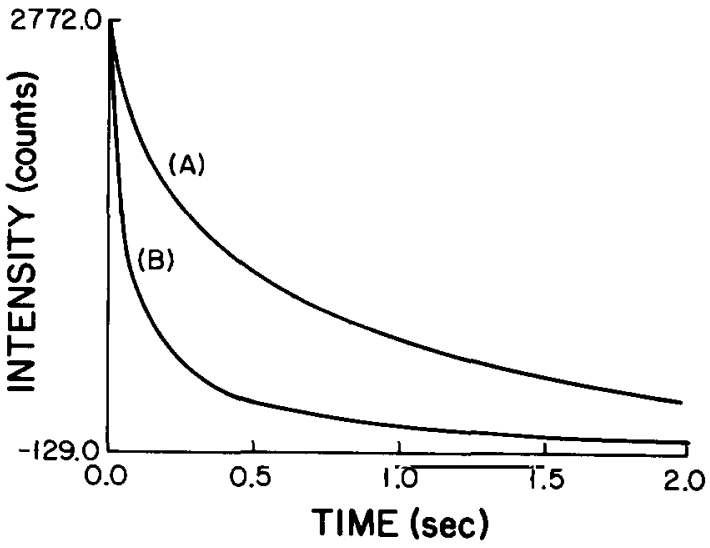

Fig. 2. Decay rates for naphthalene system (nongeminate). Curve $A$ represents steady state excitation and curve $B$ represents pulse excitation.

rates do not depend on the duration of the excitation (pulse length). This is shown in fig. 1. However, the situation is different for nongeminate recombination. Since most of the triplets in naphthalene are produced via intersystem crossing (a random process), the density in this case will depend on the duration of the excitation. This is shown in fig. 2 .

\section{Conclusions}

In our study we find that: (1) the geminate recombination decay rates do not depend on the duration of the excitation; (2) the nongeminate recombination decay rates do depend on the duration of the excitation; (3) our work provides a new dynamic technique to differentiate between geminate and nongeminate recombination kinetics of triplet excitons.

This research was supported in part by NSF Grant No. DMR 8801120 and in part by the Petroleum Research Fund administered by the American Chemical Society Grant No. 18791-AC5,6.

[1] R. Kopelman and S. Parus, J. Prasad, Chem. Phys. 128 (1988) 209.

[2] S. Parus and R. Kopelman, these Proceedings (DPC '89) p. 43.

[3] R. Kopelman, Science 241 (1988) 1620.

[4] G.T. Wright, Proc. Phys. Soc. (London) B68 (1955) 241.

[5] E.J. Bowen, E. Mikiewicz and F.W. Smith, Proc. Phys. Soc. (London) A62 (1949) 26.

[6] N.E. Geacintov, M. Pope and F.E. Vogel, Phys. Rev. Lett. $22(1969) 593$.

[7] S. Arnold, R.R. Alfano, M. Pope, W. Yu, P. Ho, R. Selsby, J. Tharrats and C.E. Swenberg, J. Chem. Phys. 64 (1976) 5104.

[8] J. Prasad and R. Kopelman, Phys. Rev. Lett. 59 (1987) 2103. 